EMBRYARIDDLE
Aeronautical University

SCHOLARLY COMMONS
International Journal of Aviation, Aeronautics, and Aerospace

\title{
Hazards Associated with Human Machine Interface in Part 121 Operations Based on ASRS Reports
}

\author{
Brian T. Musselman \\ Embry-Riddle Aeronautical University, moose@me.com
}

Follow this and additional works at: https://commons.erau.edu/ijaaa

\section{Scholarly Commons Citation}

Musselman, B. T. (2019). Hazards Associated with Human Machine Interface in Part 121 Operations Based on ASRS Reports. International Journal of Aviation, Aeronautics, and Aerospace, 6(5). https://doi.org/10.15394/ijaaa.2019.1420

This Article is brought to you for free and open access by the Journals at Scholarly Commons. It has been accepted for inclusion in International Journal of Aviation, Aeronautics, and Aerospace by an authorized administrator of Scholarly Commons. For more information, please contact commons@erau.edu. 


\section{Background}

\section{Introduction}

There are four components of a safety management system: safety policy and objectives, safety risk management, safety assurance, and safety promotion. This paper focuses on safety risk management. Hazard identification is the first step of safety risk management. In order to mitigate risk, one must first understand the hazard (International Civil Aviation Organization [ICAO], 2018; Stolzer, Halford, \& Goglia, 2011).

Poor human-machine interaction can sometimes end with catastrophic results. The Lion Air and Ethiopian Air 737 Max crashes, as a result of anomalies with the flight control software of the Maneuvering Characteristics Augmentation System, are a current example of these catastrophic results (Gitlin, 2019). These accidents were the result of a human-machine interface hazard, which was presumably not understood by the aviation industry.

\section{Problem Statement}

Airline operators functioning under Federal Aviation Administration Part 121 are required to execute a safety management system. Part of an effective safety management system is understanding (and mitigating) hazards (Federal Aviation Administration, 2015). Assuming the Boeing 737 Max hazard is not understood by the industry, then what other human-machine interface hazards could exist within Part 121 operations?

\section{Purpose of the Study}

The purpose of this study is to identify human-machine interface hazards, which lead to poor performance. Once these human-machine interface hazards in Part 121 operations are identified and understood, these hazards can be mitigated.

\section{Research Question}

This research seeks to discover what common hazards, if any, lead to poor performance as a result of human-machine interface anomalies as identified in Aviation Safety Reporting System (ASRS) Part 121 reports.

\section{Delimitations}

The current study is delimited in several ways. First, while human-machine interface challenges can result in decreased performance on any flight, this study was limited to Part 121 operations due to the structure created by the increased regulations of Part 121 operations. Second, the data spans two years, 2017 and 2018. The study was limited to these years for project scope manageability. Finally, the coding within the qualitative analysis was limited to one researcher, however; in the future, the research can be expanded to additional coders to increase validity and measure inter-rater reliability.

\section{Limitations and Assumptions}

The results are limited to Part 121 operations, and cannot be generalized to other flying operations. The author assumes accuracy of the information submitted 
by the ASRS reporter. The ASRS reports are voluntary, therefore, they cannot be used for consideration of prevalence of occurrence, and reporting bias exists because who and when personnel report is indeterminant; the power of the reports is in qualitative analysis, which supports the use of the reports for this study.

\section{Literature Review}

Humans' interaction with other humans does not always result in optimum performance. The same holds true for humans interacting with machines.

The benefits anticipated by designers and policy makers when implementing automation-increased efficiency, improved safety, enhanced flexibility of operations, lower operator workload, and so onmay not always be realized and can be offset by human performance costs associated with maladaptive use of poorly designed or inadequately trainedfor automation. (Parasuraman \& Manzey, 2010, p. 381)

Therefore, automation should increase efficiency, safety, and flexibility, and decrease workload. However, automation use can "increase workload and training requirements, decrease situational awareness, and, in extreme circumstances, lead to accidents" (Miller \& Parasuraman, 2007, p. 57). If human-machine interaction is not designed appropriately for the task, workload can increase (Cuevas, Fiore, Caldwell, \& Strater, 2007). Increases in workload result in higher operator reliance on automation irrespective of trust in automation. Categorizing increased workload and decreased situation awareness associated with automation use can be challenging, but Endsley (2017) has introduced the human-autonomy system oversight (HASO) model based on over 20-years of trust in automation and situation awareness research.

The HASO model (Figure 1) provides a construct for designing the appropriate amount of human-automation interaction into a system to support attention allocation in the performance of tasks. The HASO model is a complex interaction of multiple aspects of the human operator and the automation. Attention allocation is at the center of the HASO model. Attention allocation can be influenced by the performance of the automation, and effect the performance (situation awareness) of the operator. The HASO model provides a construct for designing the appropriate amount of human-automation interaction into a system to support attention allocation in the performance of tasks. This model can be used as a baseline for the node taxonomy. 


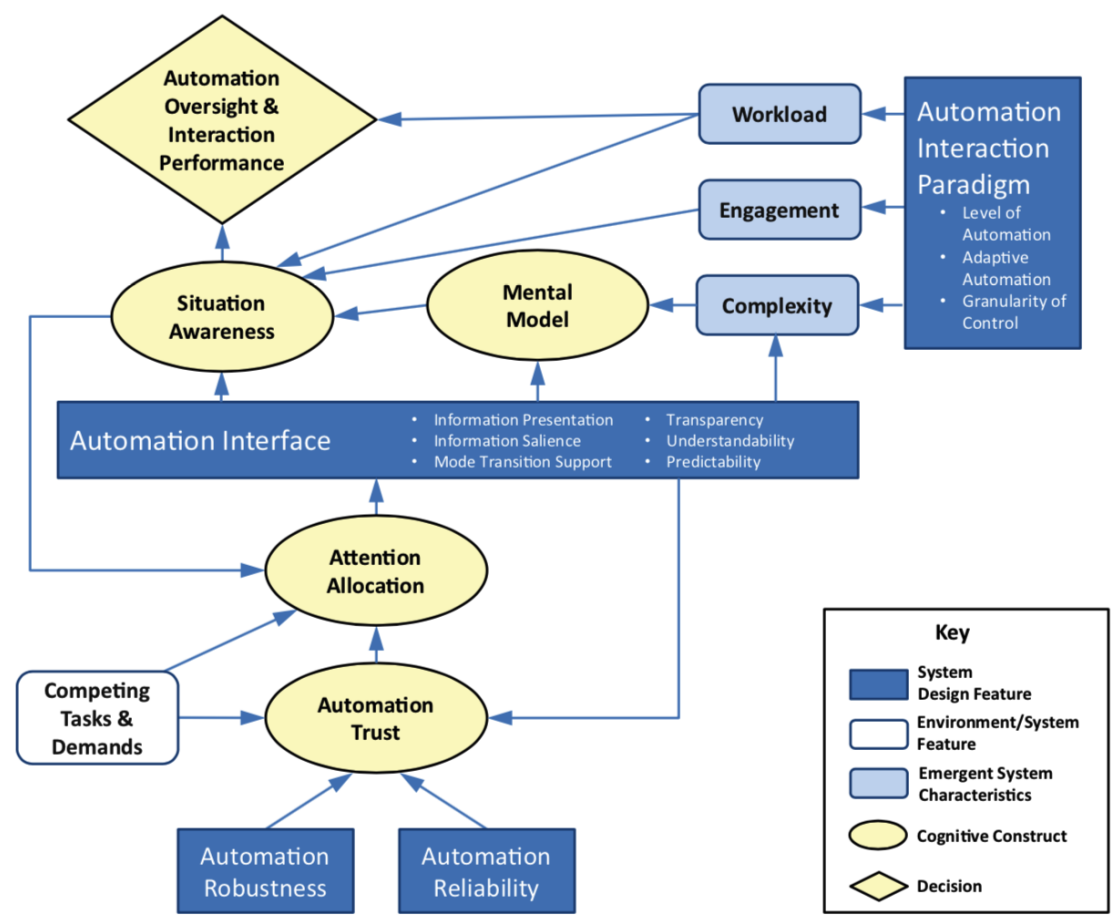

Figure 1. Human-autonomy system oversight (HASO) model (Endsley, 2017).

\section{Description of the dataset}

\section{Methodology}

The data for this research was obtained from the ASRS database. The search parameters included reports from January 2017 to December 2018 from Part 121 operations where human factors were the primary problem and human-machine interface was a reported human factor. This dataset contained 163 reports.

\section{Data Analysis Method}

The initial query of ASRS reports resulted in 163 reports. The synopsis of each of the 163 reports was reviewed to identify reports related to performance decrement due to human-machine interface. Of the 163 initial reports, 123 reports were identified as containing a performance problem related to human-machine interface. The full report narrative for each of the 123 reports was extracted from the ASRS database, and saved as separate Microsoft Word files. Each file was named as the report number. The 123 reports were imported into NVivo Plus version 12 for exploration, coding and analysis.

The first step in data exploration was to explore the dataset reports using the NVivo word frequency function. A word frequency query was executed to identify the top 100 exact matches of words four letters or longer. Four letters were used as a parameter to exclude modifier words or personal pronouns three letters or less (e.g. the, an, he, she). NVivo provides a function to exclude specific words 
from the frequency query; 'asrs' is the only word eliminated from the frequency query. All other words were applicable. The word cloud function was used to reveal prominent words to use in coding. Next, the tree map and cluster analysis functions were used to identify potential nodes for analysis. In the end, the HASO model was the primary mechanism to drive the node taxonomy (Appendix A). Situation awareness and automation oversight and interaction performance are constructs in the HASO model; however, they were not included as nodes in the taxonomy because they are the outcome (results) of the other nodes within the HASO model. This research seeks to understand the factors that contribute to the negative outcomes.

The node taxonomy at Appendix A was input into NVivo for qualitative analysis. The nodes were organized as nodes with child nodes for additional specificity within the parent node. For example, mental model was a parent node of mental model influenced by automation interface and mental model influenced by complexity. During the coding process, 19 reports were removed from the dataset because they did not contain factors associated with human-machine interface that lead to poor performance. Each of the 104 reports were coded separately using interactive coding. This is a manual coding process where the researcher highlights excerpts from the narrative, and connects this highlighted text with a node in the node taxonomy. Once all data was coded, it was available for analysis in NVivo.

\section{Results}

The dataset was analyzed various ways using NVivo. First, data analysis was accomplished using the charts function in NVivo. The charts function provides multiple means to chart the nodes and classifications. Classifications are essentially defining attributes for each of the reports. The classification attributes are month, time of day, control function (the ground control entity communicating with pilots), and flight phase. The classifications where compared with nodes in the charts function. The y-axis was identified as number of times a node was identified in the ASRS reports.

The charts function provided telling results. First, the chart function revealed a higher number of nodes coded in January and September, and a lower number in March and August (Figure 2). The number of nodes coded by time of day was unremarkable. The number of nodes coded by control function are shown in Figure 3. TRACON shows higher number of nodes. Finally, the number of nodes coded by flight phase is shown in Figure 4. Descent, approach (initial and final), and climb show the highest occurrences while taxi, landing, and parked show the lowest number of occurrences. The data displayed in Figure 5 is the same data displayed in Figure 4, but it is displayed in three dimensions. 


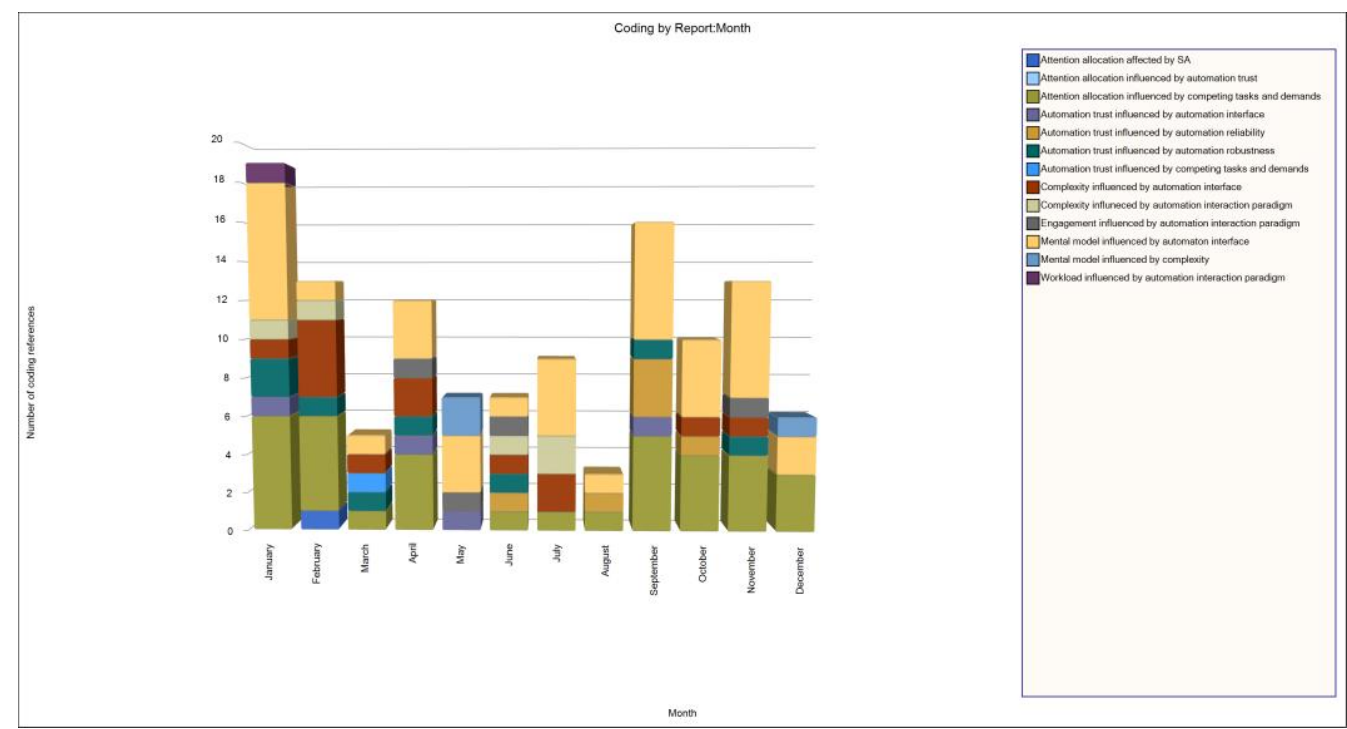

Figure 2. Nodes coded by Month.

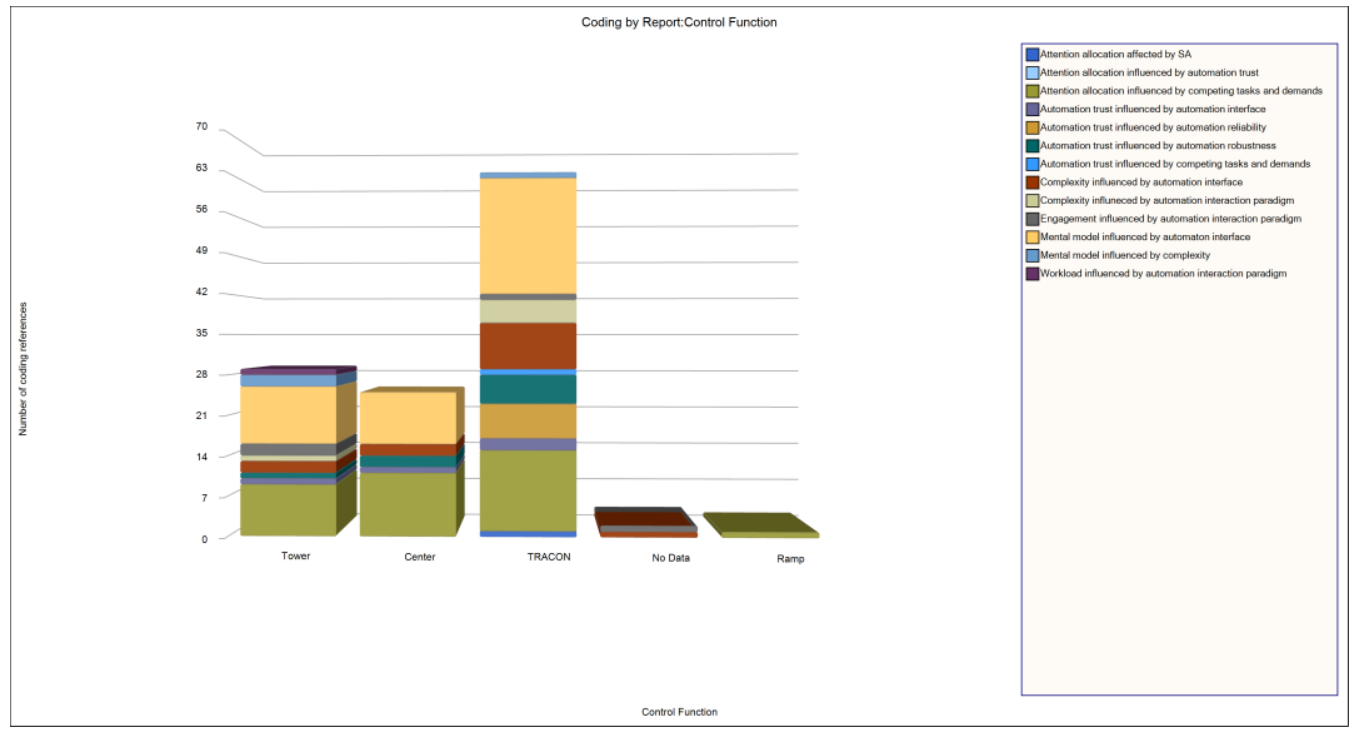

Figure 3. Nodes coded by Control Function. 
International Journal of Aviation, Aeronautics, and Aerospace, Vol. 6 [2019], Iss. 5, Art. 8

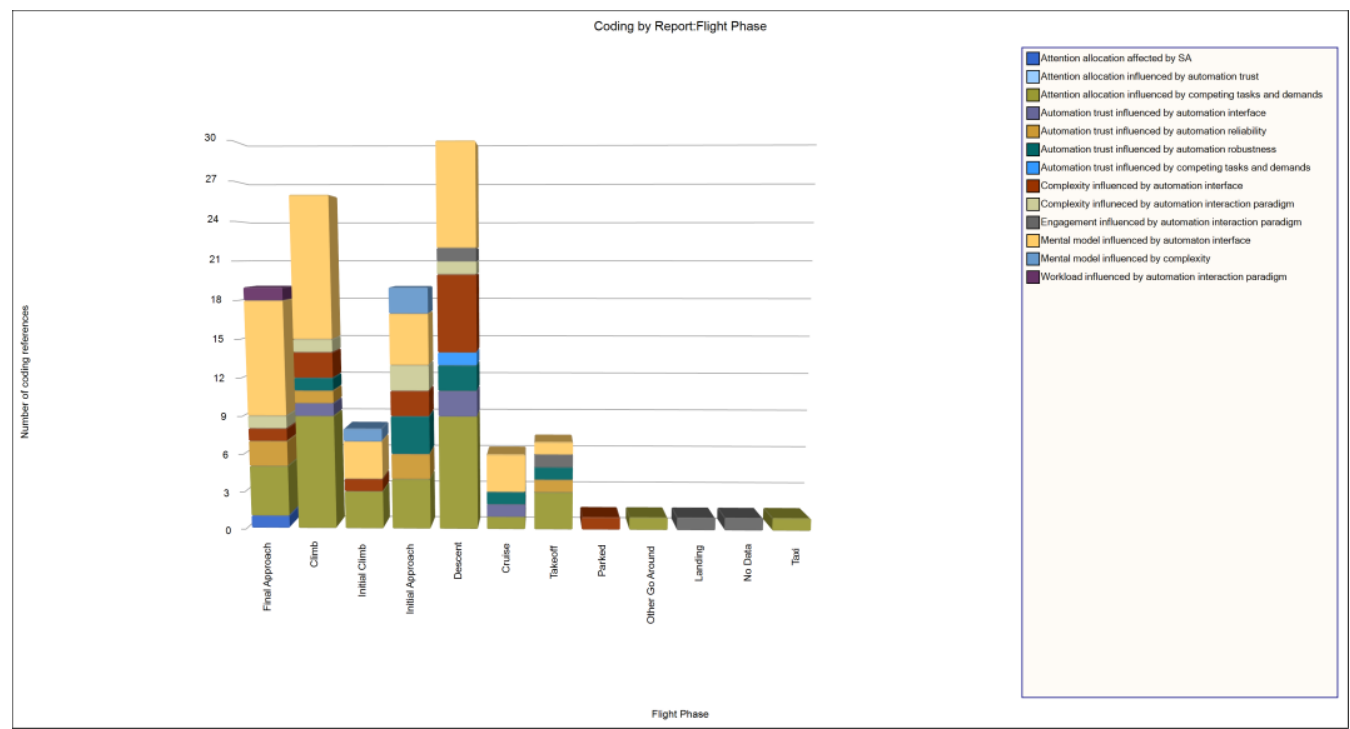

Figure 4. Nodes coded by Flight Phase (Two-Dimensional).

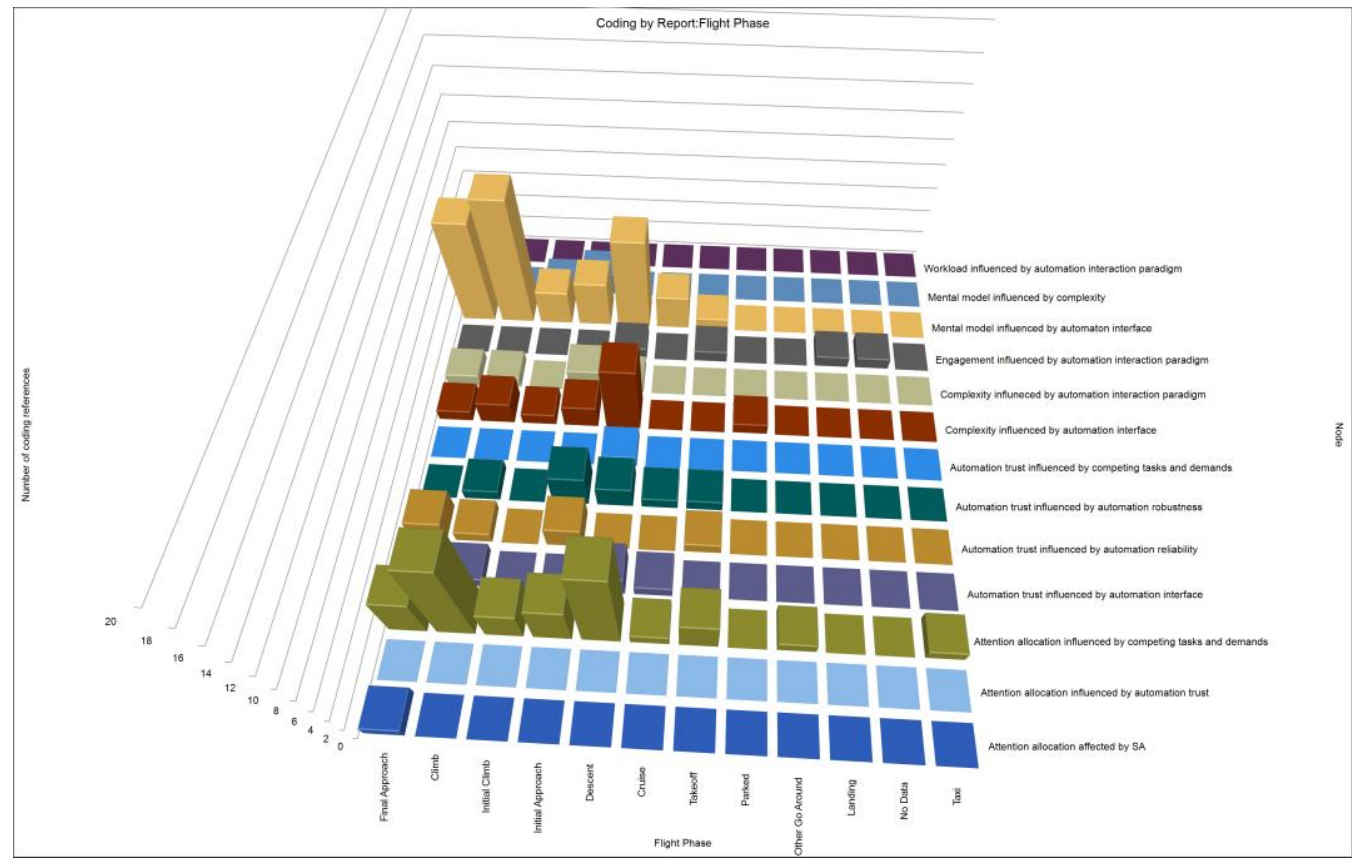

Figure 5. Nodes coded by Flight Phase (Three-Dimensional). 
Next, the hierarchy map function was used to visually display hierarchy of coding of control function to flight phase, and is shown at Figure 6. Finally, the NVivo cluster analysis function was used to help identify instances when nodes occur together, and is shown at Figure 7.

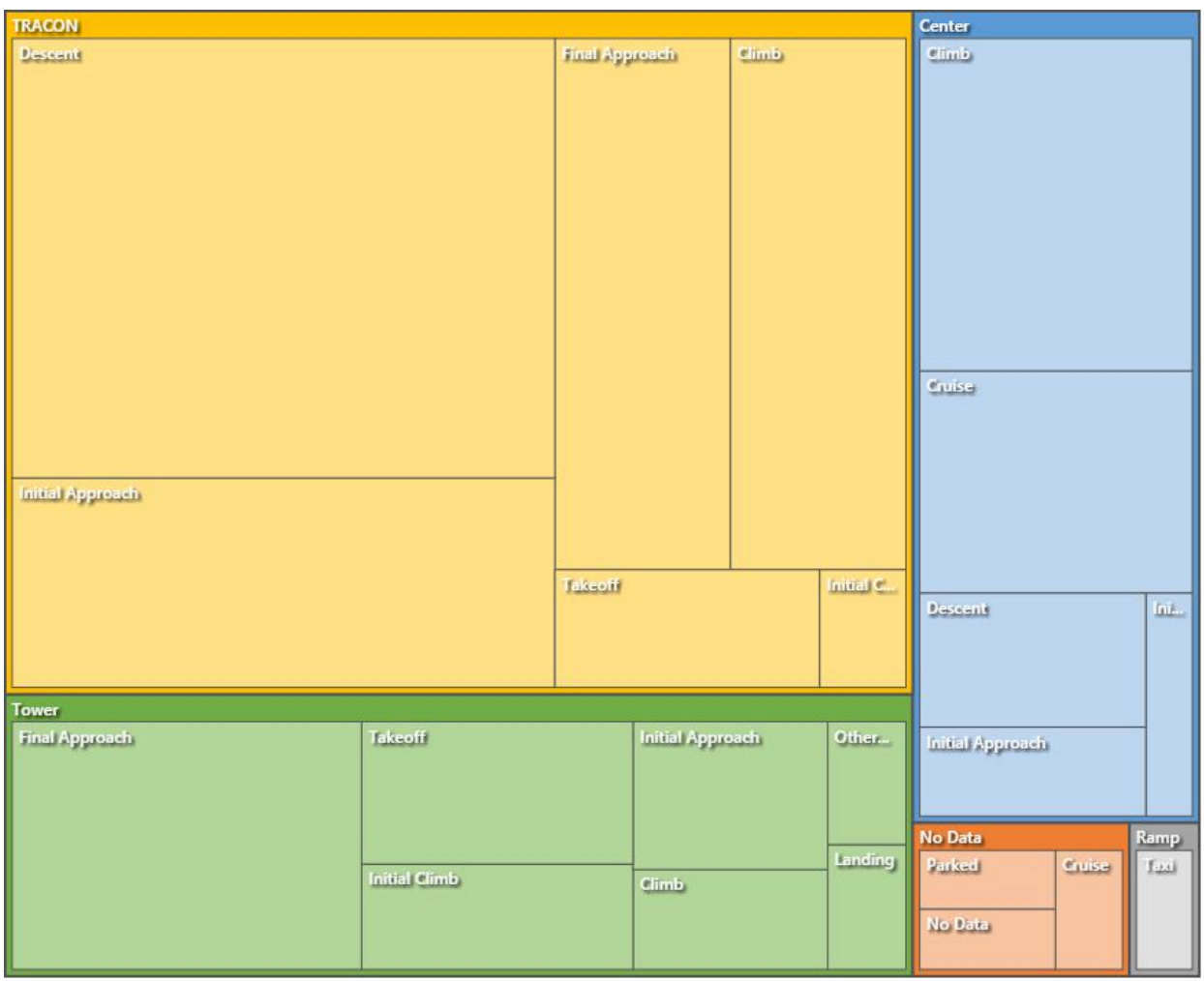

Figure 6. Hierarchy map of Flight Phase and Control Function.

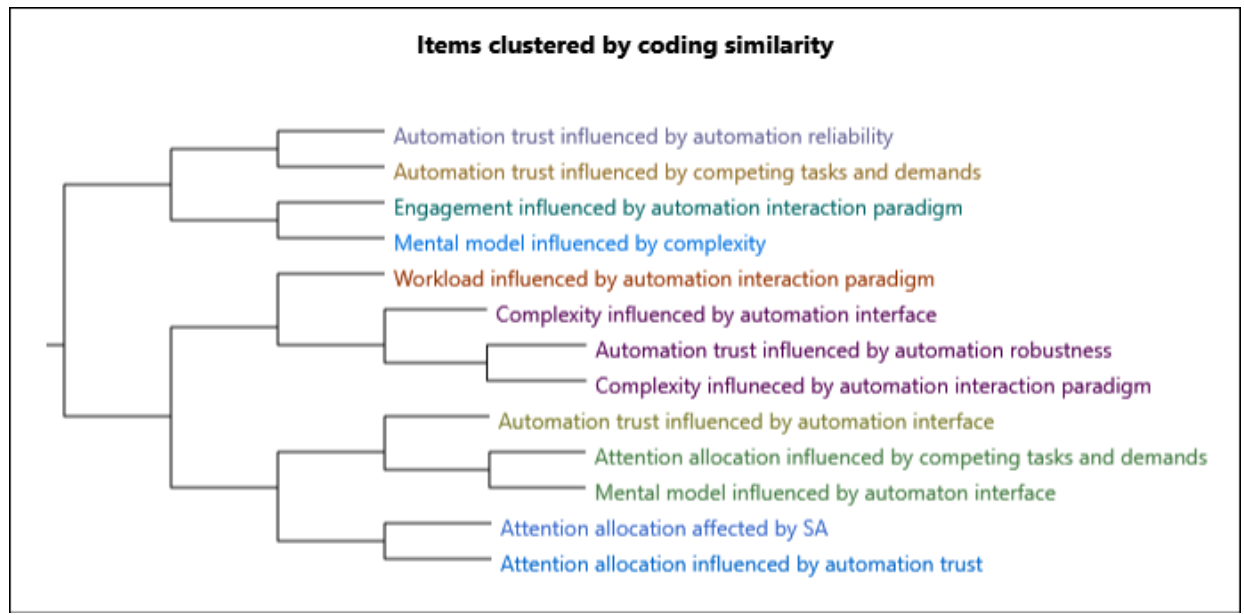

Figure 7. Nodes clustered by coding similarity. 


\section{Conclusion and Recommendations}

This qualitative study provides several remarkable results. As depicted in Figure 4 more nodes were reported in descent, approach (initial and final), and climb than other phases of flight. Descent and approach have increased humanmachine interaction requirements as the aircraft configuration is adjusted for arrival. Climb has increased human-machine interaction requirements as the aircraft configuration is adjusted for cruise flight. As expected, and shown in Figure 6 , majority of the nodes during descent and initial approach are with TRACON, during final approach are with tower, and during climb are with center.

The nodes mental model influenced by automation interface and attention allocation influenced by competing tasks and demands were coded more than any other nodes (Figure 5). Additionally, as Figure 7 shows, there is a coding similarity between these two codes. This coding similarity is evident in all phases of flight except final approach (Figure 5) where there are lower coded occurrences of automation allocation influenced by competing tasks and demands, and higher coded occurrences of mental model influenced by automation interface. However, when compared to other nodes, both nodes were still coded with higher occurrence during final approach. The high occurrence and coding similarity in these two nodes potentially identified a human-machine interaction hazard associated with poor performance. The HASO model in Figure 8 shows an associated path for these two nodes where competing tasks and demands influences attention allocation, attention allocation influences automation interface, and automation interface influences the mental model. This research identified the prevalence of these two nodes, but does not provide the granularity to understand what competing tasks and demands are influencing attention allocation or what aspects of automation interface are influencing the mental model. Further quantitative research could be performed to parse out the various aspects of automation interface and competing tasks and demands. This type of granularity can provide specific hazards of flight operations to mitigate in order to reduce poor performance and increase situation awareness. 


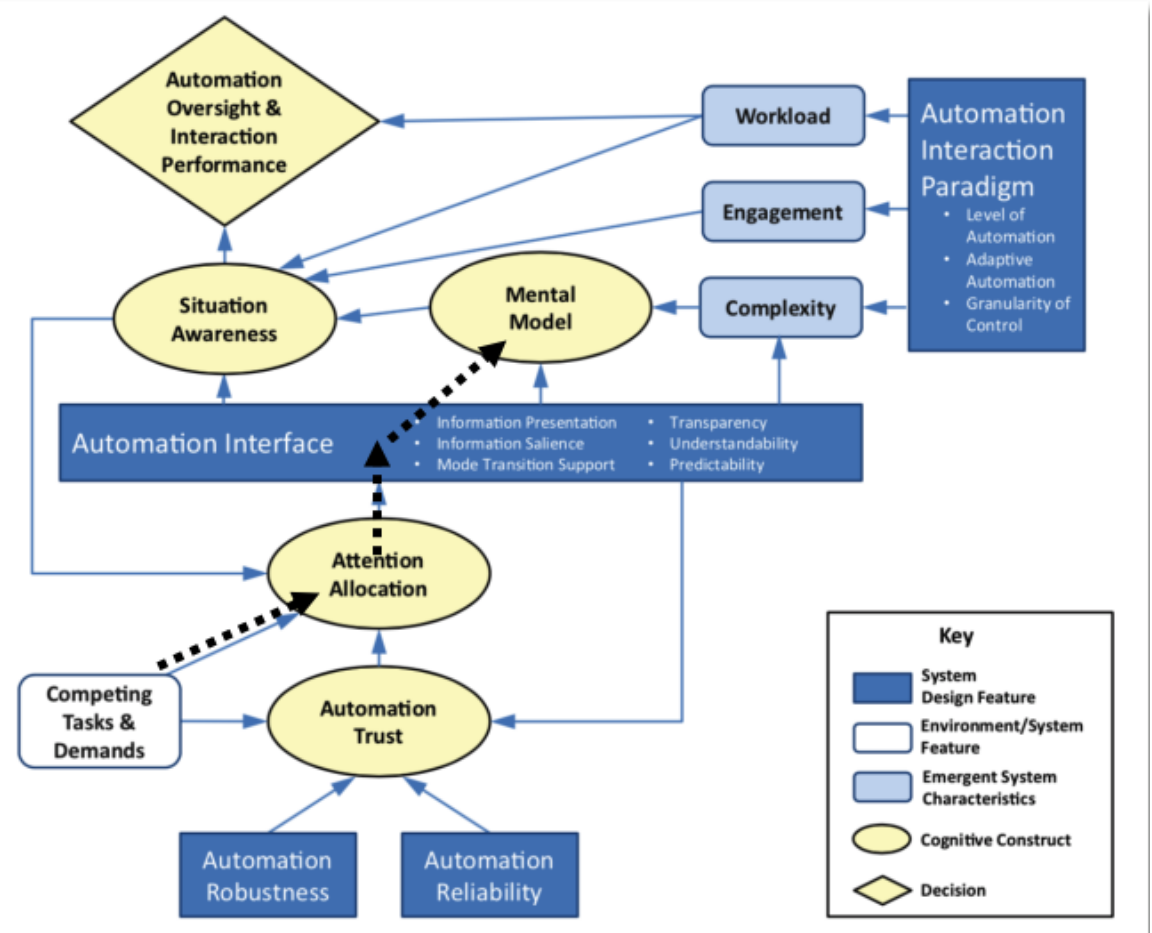

Figure 8. Human-autonomy system oversight (HASO) model with coding high occurrence and similarity identified (Endsley, 2017).

The coded occurrences of human-machine interactions in January and September are higher than other months. These results cannot be used for consideration of prevalence of occurrence; however, the difference deserves notice. A potential explanation for this occurrence could include pilot or controller work rotations, the start of pilot or controller training programs, change in weather, and other factors. It is important to conduct further research to understand the factors associated with the monthly variation of reported human-machine interaction anomalies.

This research supports the HASO model as a construct for designing the appropriate amount of human-automation interaction into a system to support attention allocation in the performance of tasks. This research identified a high prevalence and similarity of coding of mental model influenced by automation interface and attention allocation influenced by competing tasks and demands in Aviation Safety Reporting System (ASRS) Part 121 reports. Further quantitative research could be performed to parse out the various aspects of automation interface and competing tasks and demands, and to identify factors influencing human-machine interaction during different months. 


\section{References}

Cuevas, H. M., Fiore, S. M., Caldwell, B. S. \& Starter, L. (2007). Augmenting team cognition in human-automation teams performing in complex operational environments. Aviation, Space and Environmental Medicine, 78(5), 63-70.

Endsley, M. R. (2017). From here to autonomy: Lessons learned from humanautomation research. Human Factors, 59(1), 5-27.

Federal Aviation Administration. (2015). Safety management systems, 14 C.F.R. $\S$ 5. Retrieved from http://rgl.faa.gov/Regulatory_and_Guidance_Library/ rgFAR.nsf/0/3CC785F32D18CB9986257E030055D910?OpenDocument

Gitlin, J. M. (2019, June 17). "We are very sorry" Boeing division CEO apologizes for 737 Max deaths. ARSTechnica. Retrieved from https://arstechnica.com/cars/2019/06/we-are-very-sorry-boeing-ceoapologizes-for-737-max-deaths/

International Civil Aviation Organization. (2018). Safety management manual (SMM) (Doc. 9859). Montréal, Quebec, Canada: Author. Retrieved from http://dgca.gov.in/intradgca/intra/icaodocs/9859_unedited_en.pdf

Miller, C.A. \& Parasurman, R. (2007). Designing for flexible interaction between humans and automation: Delegation interfaces for supervisory control. Human Factors, 49(1), 57-75.

Parasuraman, R. (1997). Humans and automation: Use, misuse, disuse, abuse. Human Factors, 39(2), 230-253.

Parasuraman, R, \& Manzey, D. H. (2010). Complacency and bias in human use of automation: An attentional integration. Human Factors, 52(3), 381-410.

Safety management systems, 14 C.F.R. \$ 5. (2015). Retrieved from http://rgl.faa.gov/Regulatory_and_Guidance_Library/rgFAR.nsf/0/3CC785 F32D18CB9986257E030055D910?OpenDocument

Stolzer, A., Halford, C., \& Goglia, J. (2011). Implementing safety management systems in aviation. Burlington, VT: Ashgate. 


\section{Appendix A}

\section{Node Taxonomy}

Attention Allocation

- Attention allocation influenced by situation awareness

- Attention allocation influenced by automation trust

- Attention allocation influenced by competing tasks and demands

Automation Trust

- Automation trust influenced by automation interface

- Automation trust influenced by automation reliability

- Automation trust influenced by automation robustness

- Automation trust influenced by competing tasks and demands

Complexity

- Complexity influenced by automation interface

- Complexity influenced by automation interaction paradigm

Engagement influenced by automation interaction paradigm

Mental Model

- Mental model influenced by automation interface

- Mental model influenced by complexity

Workload influenced by automation interaction paradigm 